\section{Asociación entre diabetes mellitus y patología cardiovascular en la población adulta de Chile: estudio de la Encuesta Nacional de Salud 2009-2010}

\author{
ANTONIO ARTEAGA, ALBERTO MAIZ, \\ ATTILIO RIGOTTI, VÍCTOR CORTÉS
}

\section{Association between diabetes mellitus and cardiovascular disease in Chilean adults. Analysis of the national health survey 2009-2010}

Background: Diabetes mellitus (DM) is a recognized atherosclerotic cardiovascular disease (ACVD) risk factor. This association has yet to be quantified in the Chilean population. Aim: To compare the frequency of ACVD between diabetic and non-diabetic Chilean subjects. Material and Methods: Data was extracted from the Chile National Health Survey (ENS) performed in 2009-2010. $D M$ diagnosis was made with fasting glucose. ACVD (coronary, cerebral and peripheral vascular disease) was established by self-report. Major cardiovascular risk factors were identified by clinical and laboratory assessment. Results: A total of 5,416 adults (2,200 men and 3,216 women) were surveyed in ENS 20092010. Of these, 508 were diabetic and 375 reported ACVD. ACVD frequency was $16.1 \%$ and $6.1 \%$ in diabetic and non-diabetic subjects, respectively. In diabetic men, the frequency of ACVD steadily increased with age, from $5.1 \%$ to $22.1 \%$. In diabetic women, the highest frequency of ACVD (17.4\%) was found in ages ranging from 45 to 54 years. In people younger than 54 years, the odds ratio for $A C V D$ in diabetic compared to non-diabetic subjects, was 3.59 in men $\left(\chi^{2}=4.03 p<0.03\right)$ and 5.26 in women $\left(\chi^{2}=7.7 p<0.007\right)$. Cardiovascular risk factors and metabolic syndrome were significantly more common in diabetic subjects with reported ACVD. Conclusions: DM is associated with an increased frequency of ACVD and cardiovascular risk factors in Chilean adults. In line with international reports, our findings suggest that DM is also a cardiovascular risk factor in Chile, particularly relevant for women.

(Rev Med Chile 2014; 142: 175-183)

Key words: Atherosclerosis; Diabetes mellitus; Risk factors.

\author{
Departamento de Nutrición, \\ Diabetes y Metabolismo. \\ Facultad de Medicina, Pontificia \\ Universidad Católica de Chile. \\ Santiago de Chile. \\ Recibido el 18 de enero de \\ 2013, aceptado el 24 de enero \\ de 2014.

\section{Trabajo financiado por FONDECYT, Proyectos No 11100168 (V.C.) y 1110712 (A.R.)} \\ Conflictos de intereses: ninguno \\ que declarar.

Correspondencia a:
Dr. Antonio Arteaga
Marcoleta 367, Departamento
de Nutrición, Diabetes y
Metabolismo, Santiago, Chile.
Teléfono: 56223543862.
aarteaga@med.puc.cl \\ Correspondencia a: \\ Dr. Antonio Arteaga \\ Marcoleta 367, Departamento \\ de Nutrición, Diabetes y \\ Teléfono: 56223543862. \\ aarteaga@med.puc.cl
}

L a diabetes mellitus (DM) es uno de los principales problemas de salud mundial. Se estima que en el año 2004, aproximadamente 3,4 millones de personas fallecieron a raíz de sus complicaciones ${ }^{1}$ y se prevé que su prevalencia aumentará significativamente en los próximos años. En Chile, según la Encuesta
Nacional de Salud (ENS), la prevalencia de DM habría aumentado de 6,3\% en el año 2003 a 9,4\% en el 2010.

Clásicamente, el manejo clínico de la DM se ha centrado en el control glicémico y en el de las complicaciones microvasculares; sin embargo, existe consenso en que la enfermedad cardiovas- 
cular aterosclerótica (ECVA) es la primera causa de mortalidad en pacientes diabéticos (PD) 2 .

No obstante lo anterior, existen importantes diferencias en la prevalencias reportadas de ECVA en PD. La mayor parte de los datos de prevalencia de ECVA en PD proviene de estudios de certificación clínica de causa de muerte ${ }^{3}$. En el año 2004 en Estados Unidos de Norteamérica, la enfermedad coronaria fue consignada en $68 \%$ de los certificados de defunción de los PD mayores de 65 años, mientras que el accidente cerebrovascular lo fue en $16 \%$ de estos enfermos ${ }^{4}$.

La prevalencia de ECVA en PD también ha sido estimada a partir de estudios prospectivos de cohortes y de estudios observacionales transversales en poblaciones de sobrevivientes ${ }^{5-9}$. En ellos, la ECVA ha sido diagnosticada con metodologías muy diversas, incluyendo determinaciones cuantitativas de las lesiones ateroscleróticas, autoreporte de patología o sintomatología cardiovascular, estimación de riesgo de patología vascular en base de la integración de factores de riesgo mayores en modelos matemáticos. Esta heterogeneidad metodológica ha impedido comparar los estudios disponibles, determinando que la relevancia de la DM como factor de riesgo cardiovascular aún sea motivo de discrepancia entre los expertos, sobretodo en relación a su impacto diferencial en distintos grupos poblacionales clásicamente sub representados en estudios clínicos, tales como mujeres o determinados grupos étnicos.

En Chile, la prevalencia de ECVA en PD permanece desconocida. En un grupo de $368 \mathrm{PD}$ fallecidos y analizados con necropsia completa entre los años 1959 y 1983 , se encontró que 31,6\% de estos enfermos fallecieron efectivamente por ECVA (Arteaga y cols., datos no publicados).

El objetivo de este estudio fue recopilar datos actualizados representativos de la población adulta de Chile (ENS 2009-2010), acerca de la frecuencia de ECVA en PD y compararla con la de pacientes no diabéticos (PND) y con factores clásicos de riesgo cardiovascular.

\section{Materiales y Métodos}

\section{Diseño general, población y mediciones:}

Los datos utilizados en este estudio fueron extraídos de la Encuesta Nacional de Salud (ENS) 2009-2010 ${ }^{10}$, cuyo diseño fue de tipo observacional de corte transversal. En esta encuesta se reclutaron 5.416 personas voluntarias, representativas de la población general rural y urbana de Chile, de ambos sexos y mayor a 15 años de edad. La metodología empleada en la generación de esta muestra está descrita in extenso en otra publicación ${ }^{10}$.

Los participantes fueron evaluados en sus hogares por enfermeras entrenadas, quienes determinaron la presión arterial y diversos parámetros antropométricos. Además, se obtuvieron alícuotas de sangre y orina, en las que se midieron perfil bioquímico y glicemia, y microalbuminuria y creatininuria, respectivamente. Encuestadores entrenados recopilaron datos sobre síntomas y signos de ECVA, así como autoreporte de diagnóstico médico de ECVA en cualquiera de sus formas (coronaria, encefálica o periférica).

La circunferencia de cintura (CC) fue medida en la línea axilar media en el punto medio entre reborde costal y cresta ilíaca, con huincha plástica no rígida especialmente diseñada para esta encuesta. Ninguna huincha se utilizó en más de 80 determinaciones sucesivas.

La presión arterial fue evaluada con un aparato automatizado (OMRON-HEM 713C), efectuándose 2 mediciones en la misma visita, separadas por $2 \mathrm{~min}$, bajo las siguientes condiciones: horario matinal, 5 min de reposo previo, ayuno y vaciamiento vesical previo.

Los exámenes de laboratorio fueron analizados en el Laboratorio Central de la Pontificia Universidad Católica de Chile (PUC).

Para el análisis de los factores de riesgo cardiovascular se utilizó una submuestra de sujetos que disponía de mediciones de lípidos sanguíneos $(\mathrm{n}=2.642)$.

\section{Definición de las variables estudiadas:}

1. Diabetes mellitus (DM): Glicemia $\geq 126 \mathrm{mg} / \mathrm{dl}$ con ayuno $\geq 8$ h o auto reporte de diagnóstico médico de DM. Se excluyeron las pacientes con diagnóstico de DM gestacional.

2. Enfermedad cardiovascular aterosclerótica (ECVA): se consideraron diagnósticas las siguientes alternativas: a) cuestionario Rose abreviado para determinar angina de esfuerzo, b) auto reporte de diagnóstico médico de infarto agudo al miocardio, cardiopatía coronaria, accidente cerebro vascular o enfermedad vascular periférica.

3. Hipertensión arterial crónica (HTA): presión 
arterial promedio de 2 mediciones $\geq 140 / 90$ $\mathrm{mmHg}$ o autoreporte de tratamiento farmacológico de HTA.

4. Dislipidemia: a) colesterol (C)-HDL disminuido: $<40 \mathrm{mg} / \mathrm{dl}$ en hombres $\mathrm{y}<50 \mathrm{mg} / \mathrm{dl}$ en mujeres, b) hipertrigliceridemia: triglicéridos (TG) de ayuno $\geq 150 \mathrm{mg} / \mathrm{dl}, \mathrm{c}$ ) C-LDL elevado: concentración plasmática mayor a la recomendada según el riesgo cardiovascular individual calculado por tablas de Framingham, o autoreporte de tratamiento farmacológico.

5. Peso corporal: a) normal: índice de masa corporal $\left(\mathrm{IMC}\right.$ en $\left.\mathrm{kg} / \mathrm{m}^{2}\right) \geq 18,5 \mathrm{y}<24,9$; b) sobrepeso: IMC $\geq 25 \mathrm{y}<30$; c) obesidad IMC $\geq 30$; d) obesidad mórbida: IMC $\geq 40$; e) bajo peso: IMC $<18,5$.

6. Obesidad central: circunferencia de cintura (CC) $>87 \mathrm{~cm}$ hombres y $>82 \mathrm{~cm}$ mujeres

7. Tabaquismo: Fumador actual diario de al menos 1 cigarrillo al día.

8. Sedentarismo: actividad física, en tiempo libre (no laboral) de menos de $30 \mathrm{~min} 3$ veces a la semana.

9. Síndrome metabólico (SM): 3 o más de 5 criterios definidos en ATP III Update 2004 ${ }^{11}$.

10. Microalbuminuria (MA): entre $30-300 \mathrm{mg} / \mathrm{mg}$ creatinina.

\section{Análisis estadístico}

Se realizó sobre la muestra cruda no expandida de la ENS 2009-2010. En la definición de ECVA se decidió tomar los tres compromisos territoriales (coronario, encefálico y periférico) para mejorar la potencia estadística del análisis. En un modelo de contingencia de exposición-enfermedad de diseño 2 x 2 , se estimó la razón de chance (OR), su rango de distribución (intervalo de confianza 95\%) y significación estadística.

En la encuesta original existe omisión de ciertas determinaciones para algunos sujetos, por lo tanto, en el presente análisis se utilizaron las tasas corregidas por estas omisiones. De manera relevante, estas omisiones se distribuyeron aleatoriamente en los distintos grupos estudiados. Así, en algunas tablas presentadas los totales no corresponden a las sumas simples de los grupos individuales. La significación de las diferencias de las tasas observadas entre categorías se estimó con la estadística de $\chi^{2}$. Para el análisis estadístico se utilizó el programa computacional para datos tabulados y categorizados EPIDAT 3.1.

\section{Resultados}

Se analizaron 5.416 personas en total: 2.200 hombres $(40,6 \%)$ y 3.216 mujeres $(59,4 \%)$. Todos fueron mayores de 15 años de edad y provinieron de comunidades urbanas y rurales estadísticamente representativos de la población de Chile (Tabla 1). La frecuencia de DM fue de $9,4 \%$ en la muestra total, y $9,2 \%$ y $9,5 \%$ en hombres y mujeres, respectivamente. La frecuencia de ECVA (coronaria, encefálica y periférica, combinadas) fue de $6,9 \%$ en total, y de $7,7 \%$ en hombres y $6,4 \%$ en mujeres (Tabla 1).

Al comparar la proporción de personas con y sin ECVA en sujetos con y sin DM, se encontró que los $\mathrm{PD}$ tuvieron 2,7 veces más casos de ECVA que los PND (16,1\% vs 6,1\%, respectivamente) (Tabla 2). Al categorizar a los sujetos por edad, se observó que la frecuencia de PD con ECVA se incrementó progresivamente en los hombres, yendo de 5,1\% en los menores de 45 años hasta $22,1 \%$ en los mayores de 65 años (Figura 1). En mujeres con DM, en cambio, se observó frecuencia incrementada de ECVA sólo en las categorías etarias de 45 a 54 años

Tabla 1. Características generales de la población estudiada de la Encuesta Nacional de Salud (ENS) 2009-2010

\begin{tabular}{|lrrrrrr|}
\hline & \multicolumn{1}{c}{ Hombres } & \multicolumn{2}{c}{ Mujeres } & \multicolumn{2}{c|}{ Totales } \\
& n & \multicolumn{1}{c}{$\%$} & \multicolumn{1}{c}{$\mathbf{n}$} & \multicolumn{1}{c}{$\%$} & \multicolumn{1}{c|}{$\mathbf{n}$} & $\%$ \\
& 2.200 & 40,6 & 3.216 & 59,4 & 5.416 & 100,0 \\
Grupos etarios & & & & & & \\
(años) & & & & & & \\
$<45$ & 1.068 & 48,5 & 1.503 & 46,7 & 2.571 & \\
45-64 & 723 & 32,8 & 1.069 & 33,2 & 1.792 & \\
$65-74$ & 242 & 11,0 & 344 & 10,6 & 586 & \\
$>75$ & 167 & 7,7 & 200 & 9,5 & 467 & \\
Totales & 2.200 & 100,0 & 3.216 & 100,0 & 5.416 & \\
Peso corporal & & & & & & \\
Normal & 264 & 29,8 & 810 & 28,9 & 1.374 & \\
Enflaquecido & 22 & 1,2 & 67 & 2,0 & 79 & \\
Sobrepeso & 861 & 45,4 & 1.016 & 36,1 & 1.877 & \\
Obeso & 446 & 23,6 & 927 & 33,0 & 1.373 & \\
Totales* & 1.893 & 100,0 & 2.810 & 100,0 & 4.786 & \\
DM & 203 & 9,2 & 305 & 9,5 & 508 & 9,4 \\
ECVA & 168 & 7,7 & 206 & 6,4 & 375 & 6,9 \\
\hline
\end{tabular}

Abreviaciones: DM, diabetes mellitus. ECVA, enfermedad cardiovascular aterosclerótica. 


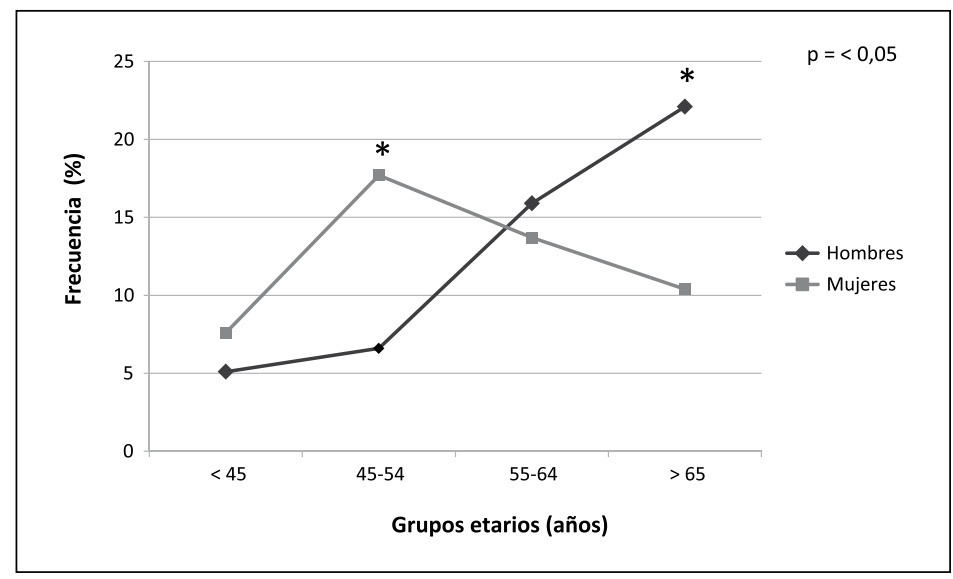

Figura 1. Frecuencia de patología cardiovascular en una población adulta de diabéticos sobrevivientes chilenos, según sexo y grupo etario. En una muestra de 508 sujetos diabéticos representativos de la población adulta de Chile, se determinó la frecuencia de enfermedad cardiovascular aterosclerótica por medio de autorreporte (ver sección de Métodos). ${ }^{*} p<0,05$ test $\chi^{2}$. y 55 a 64 años, respecto de las menores de 45 años (Figura 1). En las diabéticas mayores de 65 años, la frecuencia de ECVA no fue estadísticamente diferente que en las menores de 45 años.

En personas menores de 54 años, la razón de chance (OR, odds ratio) de ECVA en sujetos con y sin DM y fue de 3,59 en hombres (IC 95\%: 1,0213,$47 ; \chi^{2}=4,03 ; \mathrm{p}<0,03$ ) y de 5,26 en mujeres (IC 95\%: $\left.1,46-18,93 \cdot \chi^{2}=7,7 ; \mathrm{p}<0,008\right)$. En personas mayores de 55 años las diferencias no fueron estadísticamente significativas (Tabla 3 ).

Respecto a la frecuencia de factores clásicos de riesgo cardiovascular, HTA, C-LDL elevado, C-HDL bajo, triglicéridos altos, obesidad central, sedentarismo y síndrome metabólico fueron significativamente más frecuentes en PD con ECVA que en PD sin ECVA (Figura 2). Por el contrario, tanto la frecuencia de sedentarismo como de tabaquismo fue mayor en PD sin ECVA que en PD con ECVA (Figura 2). Al comparar en hombres y mujeres diabéticos, con y sin ECVA, la frecuencia de factores de riesgo cardiovascular, se encontró que sólo los triglicéridos elevados y el C-HDL bajo fueron significativamente mayores en mujeres (Figura 3). Por el contrario, en PND sólo se observó una leve pero significativa mayor frecuencia de C-HDL bajo, triglicéridos altos en mujeres (datos no mostrados). En cambio, al comparar en hombres y mujeres diabéticos con ECVA, se encontró que el colesterol total, triglicéridos elevados y el colesterol HDL bajo, fueron significativamente más frecuentes en mujeres, mientras que el sedentarismo fue significativamente menos frecuente en ellas (Tabla 4).
Tabla 2. Número y proporción de sujetos con y sin diabetes mellitus que presentan patologías cardiovasculares en ENS 2009-2010.

\begin{tabular}{|lcccccc|} 
& \multicolumn{2}{c}{ Con ECVA } & \multicolumn{2}{c|}{ Sin ECVA } & \multicolumn{2}{c|}{ Totales } \\
& n & \% & n & \% & \multicolumn{1}{c|}{$\mathbf{n}$} & \% \\
Con DM & 82 & 16,1 & 426 & 83,9 & 508 & 100 \\
Sin DM & 293 & 6,1 & 4.479 & 93,9 & 4.772 & 100 \\
Totales & 375 & & 4.905 & & 5.280 & \\
\hline
\end{tabular}

Abreviaciones: DM, diabetes mellitus. ECVA, enfermedad cardiovascular aterosclerótica

Tabla 3. Frecuencia y razón de chance (OR) de ECVA en sujetos con o sin DM, según rango etario y sexo, en ENS 2009-2010

\begin{tabular}{|c|c|c|c|}
\hline $\begin{array}{l}\text { Grupo etarios } \\
\text { años }\end{array}$ & $\begin{array}{c}\text { Con DM } \\
(\%)\end{array}$ & $\begin{array}{c}\text { Sin DM } \\
(\%)\end{array}$ & I OR (IC $95 \%$ ) \\
\hline \multicolumn{4}{|l|}{ Hombres } \\
\hline$\leq 54$ & 10,0 & 3,1 & $\begin{array}{l}3,59(1,02-13,4) \\
\chi^{2}=4,03 p<0,03\end{array}$ \\
\hline$\geq 55$ & 21,2 & 15,3 & $\begin{array}{l}1,5(0,715-3,12) \\
\chi^{2}=1,21 p<0,2\end{array}$ \\
\hline $\mathrm{n}$ de casos & 215 & 1.675 & \\
\hline \multicolumn{4}{|l|}{ Mujeres } \\
\hline$\leq 54$ & 14,3 & 2,7 & $\begin{array}{l}5,26(1,46-18,93) \\
\chi^{2}=7,7 p<0,008\end{array}$ \\
\hline$\geq 55$ & 21,6 & 12,8 & $\begin{array}{l}1,88(0,89-3,99) \\
\chi^{2} 2,83 p<0,07\end{array}$ \\
\hline $\mathrm{n}$ de casos & 326 & 2.479 & \\
\hline
\end{tabular}

Abreviaciones: DM, diabetes mellitus. ECVA, enfermedad cardiovascular aterosclerótica. OR, odds ratio. IC, intervalo de confianza. 

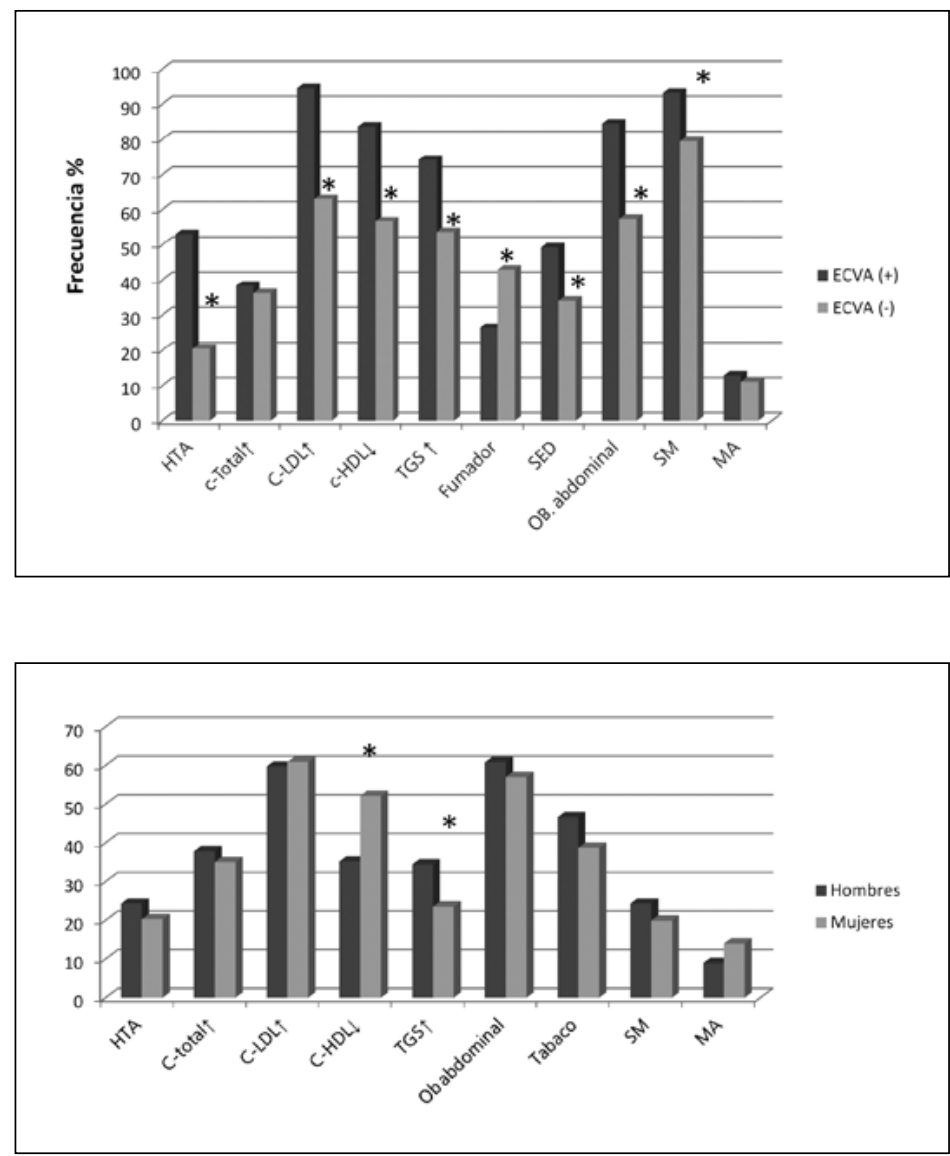

Figura 2. Frecuencia de factores de riesgo cardiovascular en una población de diabéticos chilenos con y sin patología cardiovascular. En una muestra de 508 sujetos diabéticos con y sin enfermedad vascular aterosclerótica (ECVA), representativos de la población adulta de Chile, se determinó la frecuencia de factores de riesgo cardiovascular. ${ }^{*} p<$ 0,05 test $\chi^{2}$. Para las abreviaciones referirse a la sección de Métodos.

Figura 3. Frecuencia de factores de riesgo cardiovascular en una población de hombres y mujeres diabéticos chilenos. En una muestra de 508 pacientes diabéticos, considerando tanto sujetos con y sin ECVA, se determinó la frecuencia de factores de riesgo cardiovascular en hombres y mujeres. ${ }^{*} \mathrm{p}<$ 0,05 test $\chi^{2}$. Para las abreviaciones referirse a la sección de Métodos.

Tabla 4. Diferencia de la frecuencia de factores de riesgos cardiovasculares (\%) entre hombres y mujeres diabéticos, con y sin patología cardiovascular. Población adulta Encuesta Nacional de salud 2009-2010

\begin{tabular}{|lcccccccc|}
\hline $\begin{array}{l}\text { Factores } \\
\text { de Riesgo }\end{array}$ & ECVA (+) & $\begin{array}{c}\text { Mujeres } \\
\text { ECVA (-) }\end{array}$ & $\boldsymbol{\Delta}$ & ECVA (+) & $\begin{array}{c}\text { Hombres } \\
\text { ECVA (-) }\end{array}$ & $\boldsymbol{\Delta}$ & $\begin{array}{c}\text { Diferencia } \\
\boldsymbol{\Delta}_{\text {(Mujer-hombre) }}\end{array}$ & p \\
PA $\uparrow$ & 81,9 & 55,3 & 26,6 & 84,3 & 67,0 & 17,3 & 9,3 & \\
C-Total $\uparrow$ & 76,0 & 55,7 & 19,3 & 55,5 & 60,4 & $-4,9$ & 24,2 & $<0,0003$ \\
C-LDL $\uparrow$ & 94,7 & 64,6 & 30,1 & 94,2 & 70,2 & 24,0 & 6,1 & \\
C-HDL $\downarrow$ & 90,2 & 58,5 & 31,7 & 67,4 & 67,4 & 13,4 & 18,3 & $<0,0027$ \\
TGS $\uparrow$ & 78,7 & 53,7 & 25,0 & 62,5 & 50,4 & 12,1 & 12,9 & $<0,0288$ \\
Fumador & 14,3 & 28,2 & $-13,9$ & 12,8 & 23,8 & $-11,0$ & $-2,9$ & \\
Ob. central & 90,1 & 86,0 & 4,1 & 79,9 & 80,9 & $-1,0$ & 5,1 & \\
SED & 43,2 & 34,6 & 8,6 & 55,7 & 34,1 & 21,6 & $-13,0$ & $<0,0190$ \\
SM & 90,8 & 75,5 & 15,3 & 100,0 & 84,6 & 15,4 & $-0,1$ & \\
MA & 31,5 & 23,2 & 8,3 & 32,4 & 24,1 & 8,3 & 0,0 & \\
\hline
\end{tabular}

Abreviaciones: DM, diabetes mellitus. ECVA, enfermedad cardiovascular aterosclerótica. PA, presión arterial. C, colesterol. TGS, triglicéridos. Ob, obesidad. SED, sedentarismo. SM, síndrome metabólico. MA, microalbuminuria. 
La OR de SM fue de 3,1 (IC 95\%: 1,08-8,86; $\left.\chi^{2}=4,04 ; \mathrm{p}=0,04\right)$ en PD con ECVA respecto de los sin ECVA, implicando la presencia de al menos dos factores de riesgo cardiovasculares adicionales en estos pacientes (Tabla 5). No se encontraron diferencias significativas en la frecuencia de obesidad central o microalbuminuria en función de la presencia de ECVA.

\section{Discusión}

En el presente estudio encontramos que: 1) La frecuencia estimada de DM y ECVA compuesta fue de $9,4 \%$ y $6,9 \%$, respectivamente, en la población adulta de sobrevivientes chilenos; 2) $\mathrm{La}$ frecuencia de ECVA fue de $16,1 \%$ en PD y de 6,1\% en PND y ésta aumentó directamente con la edad en PD hombres. En PD mujeres esta tendencia creciente de ECVA sólo se observó en rangos etarios medios; 3) En menores de 54 años de edad, la OR de ECVA fue de 3,5 en PD hombre y de 5,7 en PD mujer; 4) La frecuencia de HTA, C-LDL elevado, C-HDL bajo, triglicéridos elevados, obesidad central y síndrome metabólico fue significativamente mayor, mientras que la de tabaquismo fue menor en PD con ECVA respecto a los PD sin ECVA.

Estas últimas observaciones sugieren que mientras algunos factores de riesgo cardiovascular (sedentarismo y tabaquismo) en los PD con ECVA han sido favorablemente modificados por intervenciones de promoción de salud, otros (HTA, dislipidemia aterogénica, obesidad central) han sido refractarios a estas intervenciones, posiblemente porque dependen de factores fisiopatológicos mayormente conectados con la desregulación metabólica de la diabetes. Concordantemente, en nuestro estudio 92,7\% de los PD con ECVA cumplió con criterios diagnósticos de SM, implicando la presencia de al menos dos factores de riesgo cardiovascular adicionales.

Datos similares a los encontrados por nosotros han llevado a plantear a algunos autores que la magnitud del riesgo cardiovascular conferido por la DM dependería fundamentalmente de las características del estilo de vida y la frecuencia de factores de riesgo cardiovascular ${ }^{9}$. Sin embargo, la corrección por estos factores de riesgo ha mostrado a otros investigadores que la DM por si misma explica un riesgo cardiovascular significativamente aumentado ${ }^{12}$.

En el año 1985 se publicó un estudio multina-

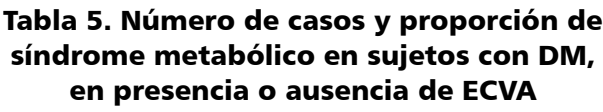

Tabla 5. Número de casos y proporción de síndrome metabólico en sujetos con DM, en presencia o ausencia de ECVA

\begin{tabular}{|lrrrrr|} 
& \multicolumn{2}{c}{ Con ECVA } & \multicolumn{2}{c}{ Sin ECVA } & Total \\
& $\mathbf{n}$ & \multicolumn{1}{c}{$\%$} & \multicolumn{1}{c|}{ n } & \multicolumn{1}{c}{$\%$} & \\
Con SM & 52 & 92,8 & 282 & 81,5 & 334 \\
Sin SM & 4 & 7,2 & 64 & 18,5 & 68 \\
Total & 56 & 100 & 346 & 100 & 382 \\
\hline
\end{tabular}

Abreviaciones: DM, diabetes mellitus. ECVA, enfermedad cardiovascular aterosclerótica. SM, síndrome metabólico. $\chi^{2}=4,04 \quad p<0,04 \quad$ OR $=3,1(95 \%$ IC 1,08-8,86).

cional de corte transversal en relación a la prevalencia de patología cardiovascular en diabéticos que incluyó 13 diferentes regiones mundiales, abarcando diversas condiciones socio-económicas-culturales poblacionales ${ }^{9}$. En este estudio, en donde, al igual que en la ENS 2009-2010, el diagnóstico de ECVA se estableció por autoreporte en 6.695 PD hombres y mujeres de 35 a 54 años de edad, se encontró que la frecuencia de ECVA global en PD varió entre 14,3 y 52,9\% y que no estuvo correlacionada con el nivel socio-económico de las regiones estudiadas. Similarmente a lo encontrado por nosotros, la ECVA fue también más frecuente en PD mujeres?.

Estudios más recientes, también de tipo observacional-transversal, han descrito prevalencias de ECVA entre 15,3 y $26,7 \%{ }^{13,14}$.

La frecuencia de ECVA en PD que reportamos en el presente estudio para población chilena $(16,1 \%)$ se encuentra en el rango inferior de estos datos internacionales, posiblemente en concordancia con la tendencia global de menor morbimortalidad cardiovascular en años recientes. Concordantemente, un estudio multicéntrico brasileño, también de tipo observacional-transversal, reportó recientemente una prevalencia global de ECVA de 21,6\% en $\mathrm{PD}^{15}$.

Por otro lado, estudios observacionales longitudinales han revelado prevalencias de ECVA en PD de entre 19,4 y $56,1 \%{ }^{16-21}$. Así, en un estudio longitudinal de 65.651 PD del país vasco, se encontró una prevalencia de patología cardiovascular de $22 \%$ en PD de diagnóstico reciente y de $33,0 \%$ en PD de larga data ${ }^{21}$.

La mayor OR de ECVA en PD mujeres respecto a PD hombres en Chile $(5,7$ vs 3,5$)$ es notoriamen- 
te coincidente con la información publicada en la versión 2012 de las Guías Europeas de Prevención Cardiovascular, en donde se describe textualmente que "individuos con DM diagnosticada tienen un incremento del riesgo cardiovascular de 5 veces en la mujer y 3 en el hombre"22.

El estudio de Framingham destacó por primera vez que en mujeres pre-menopáusicas la DM incrementaba el riesgo cardiovascular significativamente más que en hombres de igual edad ${ }^{23}$. Posteriormente, numerosos estudios poblacionales de observación han corroborado esta observación ${ }^{24-30}$.

En un meta-análisis reciente que comprendió 37 cohortes y $447.067 \mathrm{PD}$, se estimó que el riesgo de infarto miocárdico fatal y muerte cardiovascular asociado a DM fue mayor en mujeres, en todos los rangos etarios, en comparación con hombres $(3,5 \text { versus } 2,06)^{31}$.

Las causas de esta diferencia de género en la frecuencia, y posiblemente en la severidad ${ }^{27,31}$, de ECVA en PD permanecen desconocidas. De hecho, la presencia de diabetes en mujeres adelanta la edad del primer infarto miocárdico a un rango etario equivalente al de los hombres ${ }^{30,32}$.

Algunos autores señalan que este fenómeno pudiese depender mayoritariamente de la mayor carga de factores de riesgo cardiovascular en las PD mujeres, ya que las diferencias observadas, en sus estudios, se anulan al corregir por estos factores ${ }^{30}$.

Otros investigadores postulan que la mayor ECVA en PD mujeres podría atribuirse a variados factores hormonales, tales como el uso de anticoncepción oral, menopausia precoz o aparición del síndrome de ovario poliquístico ${ }^{30}$.

Es difícil explicar la caída de la frecuencia de patología coronaria en PD en los grupos etarios superiores, a diferencia de lo que sucede en el hombre. Una posible causa podría ser que en una población de sobrevivientes como la nuestra, un porcentaje de mujeres que presentaron patología cardiovascular en la pre menopausia, fallecieron, debido a la reconocida mayor letalidad de la cardiopatía coronaria que en el hombre, y posiblemente a una menor vigilancia del equipo de salud, ya que el equipo médico no estaría sensibilizado a esta problemática en la etapa reproductiva.

En el estudio poblacional de Kuopio, Finlandia, se concluyó que el mayor efecto de la DM en la mujer sobre su salud cardiovascular se podría deber al mayor impacto que tendría la hipertensión arterial y la dislipidemia aterogénica, ambas consecuencias de la resistencia insulínica, en estas pacientes ${ }^{25}$.

En resumen, nuestros hallazgos sugieren fuertemente que la DM constituye un factor de riesgo cardiovascular en la población chilena y que existe un impacto diferencial de esta enfermedad sobre la salud cardiovascular entre sexos. En las mujeres la DM determina, por mecanismos aún desconocidos, un riesgo cardiovascular excesivo en relación con los hombres.

La tendencia creciente en la incidencia de DM tanto en Chile como en el resto del mundo, hará que las patologías cardiovasculares serán un problema relevante de salud pública en el futuro próximo, particularmente en mujeres pre menopáusicas. Para abordar apropiadamente este desafío serán necesarios nuevos estudios poblacionales que permitan delimitar con mayor precisión los factores clínicos que modulan la evolución de la ECVA en PD, así como estudios básicos que permitan entender las bases fisiopatológicas de la patología aterosclerótica en DM y su mayor impacto en mujeres.

\section{Referencias}

1. Organization WH. Diabetes Mellitus World Prevalence. updated March 2013; Available from: http://www.who. int/mediacentre/factsheets/fs312/en/.

2. Buse JB, Ginsberg HN, Bakris GL, Clark NG, Costa F, Eckel R, et al. Primary prevention of cardiovascular diseases in people with diabetes mellitus: a scientific statement from the American Heart Association and the American Diabetes Association. Circulation 2007; 115 (1): 114-26.

3. Organization WH. Global Health Observatory Data Repository. Mortality: Cardiovascular diseases and diabetes, deaths per 100,000 by country. Available from: http:// apps.who.int/gho/data/node.main.A865.

4. Prevention CfDCa. National Diabetes Fact Sheet. 2011; Available from: http://www.cdc.gov/diabetes/pubs/pdf/ ndfs_2011.pdf.

5. Preis SR, Pencina MJ, Hwang SJ, D’Agostino RB, Sr., Savage PJ, Levy D, et al. Trends in cardiovascular disease risk factors in individuals with and without diabetes mellitus in the Framingham Heart Study. Circulation 2009; 120 (3): 212-20.

6. Folsom AR, Szklo M, Stevens J, Liao F, Smith R, Eckfeldt $\mathrm{JH}$. A prospective study of coronary heart disease in relation to fasting insulin, glucose, and diabetes. The 
Atherosclerosis Risk in Communities (ARIC) Study. Diabetes Care 1997; 20 (6): 935-42.

7. van Dieren S, Peelen LM, Nothlings U, van der Schouw YT, Rutten GE, Spijkerman AM, et al. External validation of the UK Prospective Diabetes Study (UKPDS) risk engine in patients with type 2 diabetes. Diabetologia 2011; 54 (2): 264-70.

8. Fox CS, Coady S, Sorlie PD, Levy D, Meigs JB, D'Agostino RB Sr, et al. Trends in cardiovascular complications of diabetes. JAMA 2004; 292 (20): 2495-9.

9. Prevalence of small vessel and large vessel disease in diabetic patients from 14 centres. The World Health Organisation Multinational Study of Vascular Disease in Diabetics. Diabetes Drafting Group. Diabetologia 1985; 28 Suppl: 615-40.

10. Encuesta Nacional de Salud 2009-2010. http://www. minsal.gob.cl/portal/url/item/bcb03d7bc28b64dfe 040010165012d23.pdf

11. Grundy SM, Brewer HB, Jr., Cleeman JI, Smith SC Jr, Lenfant C. Definition of metabolic syndrome: Report of the National Heart, Lung, and Blood Institute/American Heart Association conference on scientific issues related to definition. Circulation 2004; 109 (3): 433-8.

12. Yusuf S, Hawken S, Ounpuu S, Dans T, Avezum A, Lanas F, et al. Effect of potentially modifiable risk factors associated with myocardial infarction in 52 countries (the INTERHEART study): case-control study. Lancet 2004; 364 (9438): 937-52.

13. Avogaro A, Giorda C, Maggini M, Mannucci E, Raschetti $\mathrm{R}$, Sarli E, et al. The prevalence of coronary heart disease in Type 2 diabetic patients in Italy: the DAI study. Diabet Med 2004; 21 (7): 738-45.

14. Tran AT, Straand J, Diep LM, Meyer HE, Birkeland KI, Jenum AK. Cardiovascular disease by diabetes status in five ethnic minority groups compared to ethnic Norwegians. BMC Public Health 2011; 11: 554.

15. Gomes MB, Giannella-Neto D, Faria M, Tambascia M, Fonseca RM, Rea R, et al. Estimating cardiovascular risk in patients with type 2 diabetes: a national multicenter study in Brazil. Diabetol Metab Syndr 2009; 1 (1): 22.

16. Bueno H, Hernáez R, Hernández AV. Diabetes mellitus tipo 2 y enfermedad cardiovascular en España: una revisión descriptiva. Rev Esp Cardiol 2008; 8 (Supl. C): 53-61.

17. Reynoso-Noveron N, Mehta R, Almeda-Valdés P, RojasMartínez R, Villalpando S, Hernández-Ávila M, et al. Estimated incidence of cardiovascular complications related to type 2 diabetes in Mexico using the UKPDS outcome model and a population-based survey. Cardiovasc Diabetol 2011; 10:1.

18. Gerstein HC, Riddle MC, Kendall DM, Cohen RM,
Goland R, Feinglos- MN, et al. Glycemia treatment strategies in the action to control cardiovascular risk in diabetes (ACCORD) trial. Am J Cardiol 2007; 99 (12A): $34 \mathrm{i}-43 \mathrm{i}$.

19. Abraira C, Colwell J, Nuttall F, Sawin CT, Henderson W, Comstock JP, et al. Cardiovascular events and correlates in the Veterans Affairs Diabetes Feasibility Trial. Veterans Affairs Cooperative Study on Glycemic Control and Complications in Type II Diabetes. Arch Intern Med 1997; 157 (2): 181-8.

20. Patel A, MacMahon S, Chalmers J, Neal B, Billot L, Woodward $\mathrm{M}$, et al. Intensive blood glucose control and vascular outcomes in patients with type 2 diabetes. $\mathrm{N}$ Engl J Med 2008; 358 (24): 2560-72.

21. Arteagoitia JM, Larranaga MI, Rodríguez JL, Fernández I, Pinies JA. Incidence, prevalence and coronary heart disease risk level in known Type 2 diabetes: a sentinel practice network study in the Basque Country, Spain. Diabetologia 2003; 46 (7): 899-909.

22. Perk J, De Backer G, Gohlke H, Graham I, Reiner Z, Verschuren M, et al. European Guidelines on cardiovascular disease prevention in clinical practice (version 2012). The Fifth Joint Task Force of the European Society of Cardiology and Other Societies on Cardiovascular Disease Prevention in Clinical Practice (constituted by representatives of nine societies and by invited experts). Eur Heart J 2012; 33 (13): 1635-701.

23. Kannel WB, McGee DL. Diabetes and cardiovascular disease. The Framingham study. JAMA 1979; 241 (19): 2035-8.

24. Kanaya AM, Grady D, Barrett-Connor E. Explaining the sex difference in coronary heart disease mortality among patients with type 2 diabetes mellitus: A meta-analysis. Arch Intern Med 2002; 162 (15): 1737-45.

25. Juutilainen A, Kortelainen S, Lehto S, Ronnemaa T, Pyorala K, Laakso M. Gender difference in the impact of type 2 diabetes on coronary heart disease risk. Diabetes Care 2004; 27 (12): 2898-904.

26. Gouni-Berthold I, Berthold HK, Mantzoros CS, Böhm M, Krone W. Sex Disparities in the Treatment and Control of Cardiovascular Risk Factors in Type 2 Diabetes. Diabetes Care 2008; 31 (7): 1389-91.

27. Norhammar A, Stenestrand U, Lindbäck J, Wallentin L. Women younger than 65 years with diabetes mellitus are a high-risk group after myocardial infarction: a report from the Swedish Register of Information and Knowledge about Swedish Heart Intensive Care Admission (RIKS-HIA). Heart 2008; 94 (12): 1565-70.

28. Anand SS, Islam S, Rosengren A, Franzosi MG, Steyn K, Yusufali AH, et al. Risk factors for myocardial infarction in women and men: insights from the INTERHEART 
Diabetes mellitus y enfermedad cardiovascular en Chile - A. Arteaga et al

study. Eur Heart J 2008; 29 (7): 932-40.

29. Kramer HU, Raum E, Ruter G, Schottker B, Rothenbacher D, Rosemann T, et al. Gender disparities in diabetes and coronary heart disease medication among patients with type 2 diabetes: results from the DIANA study. Cardiovasc Diabetol 2012; 11: 88.

30. Norhammar A, Schenck-Gustafsson K. Type 2 diabetes and cardiovascular disease in women. Diabetologia
2013; 56 (1): 1-9.

31. Huxley R, Barzi F, Woodward M. Excess risk of fatal coronary heart disease associated with diabetes in men and women: meta-analysis of 37 prospective cohort studies. BMJ 2006; 332 (7533): 73-8.

32. Gu K, Cowie CC, Harris MI. Diabetes and decline in heart disease mortality in US adults. JAMA 1999; 281 (14): 1291-7. 